\title{
Features of the Use of Microprocessors in the Systems of Ovojectors in their Adaptation to the Conditions of the Former CIS
}

\author{
Murad Anver oglu Omarov \\ Department \\ of Natural Sciences \\ Kharkiv National University of Radio \\ Electronics \\ Kharkiv, Ukraine \\ murad.omarov@nure.ua
}

\author{
Volodymyr Kartashov \\ Department of Media Engineering and \\ Information Radioelectronic Systems \\ Kharkiv National University of Radio \\ Electronics \\ Kharkiv, Ukraine \\ volodymyr.kartashov@nure.ua
}

\author{
Roman Tsekhmistro \\ Department of Media Engineering and \\ Information Radioelectronic Systems \\ Kharkiv National University of Radio \\ Electronics \\ Kharkiv, Ukraine \\ tsekhmistroroman@gmail.com
}

\begin{abstract}
This electronic document describes the use of microcontrollers in the regulations of automatic sorting of eggs with a live (dead) embryo with further automatic vaccination of live embryos. These installations are produced only in 3 countries and are called Ovojectors.
\end{abstract}

Keywords-microcontroller system, light sensor, mechanical extraction, computer control

\section{INTRODUCTION}

These devices (Ovojectors) are widely used by modern poultry farms. In Russia, they are leased to 5-7 poultry farms Fig.1. However, although they are produced in three to five countries, including in France, the monopoly right belongs to the company Embrex (USA). Renting these installations costs at least $\$ 220,000$. These plants increase productivity by $8-10$ times. The introduction of the proposed device will allow [1-5]: to reduce human costs by $8-10$ times; to increase lab our productivity by 3-5 times; to reduce the percentage of defective products to the total volume by $1.5-2$ times in poultry factories with a throughput of 5,000-50,000 eggs per shift.

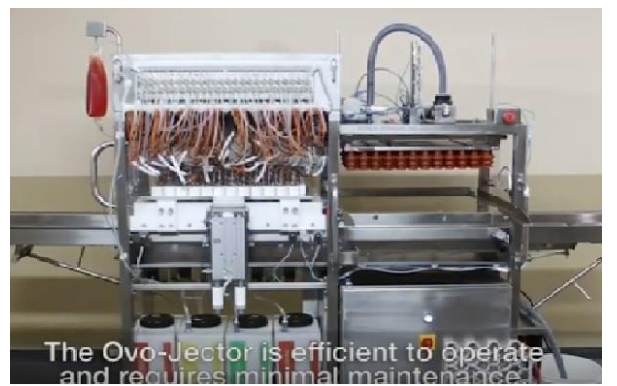

Fig. 1. Ovojector.

In ovo vaccination has been revolutionizing the avian vaccination process worldwide [6]. This is already happening in the hatcheries in Brazil and offers many technical advantages. Built with American technology and using spare parts from global suppliers fig. 2-3: OMRON photoelectricts E3F2-LS10 fig. 3; Innovation in Miniature the Lee Company Fig. 2.

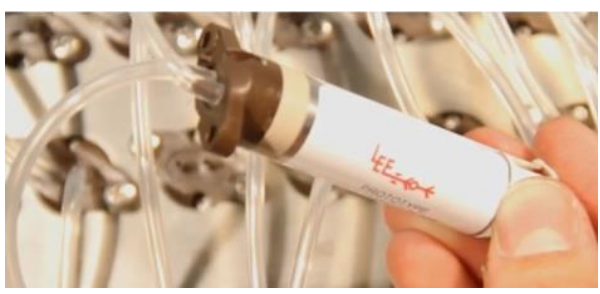

Fig. 2. Miniature the Lee Company.

The ovojectors is Boehringer inteheim's answers to in ovo vaccination. It is backed by globally structured vaccination audits, technical support, and hatchery expertise. The ovojector is efficcent to operate and requires minimal maintenance. The ovojector vaccinater is very easy to operate. The control panel is available for multi-languages. The ovojectors has a touch screen that is capable of performing self-diagnosis.

All commands can easily be performed manually, through maintename screens. Ovojectors: Efficient and simple vaccination process. Using the control panel, the operator can adjust for 3 different egg size during the transfer. Ovojectors: capable of vaccionating 35 thousand eggs per hour; the Ovojectors relies the innovative EggCheck System (Selective vaccination).

This system consist of sensors with identify the absence of eggs in egg trays during injection there by avoiding waste of vaccine.

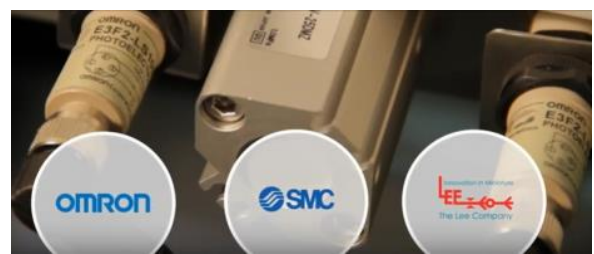

Fig. 3. Omron-electric, microcontrollers.

\section{MAIN PARTS}

These installations consist of two parts (carry out two operations): separation of a batch of eggs into parts with "live", "dead" embryos in terms of a conveyor with 
automated, appropriate consideration of the number of sorted eggs in real time Fig 2; automatic, precision dosed introduction of a vaccine into selected eggs with live embryos with the corresponding computer accounting in real time.

This report is devoted to the description of the features of the use of microcontroller equipment in tasks that are solved by the first part of installations (Ovojectors) in the CIS market, for example Fig 4 .

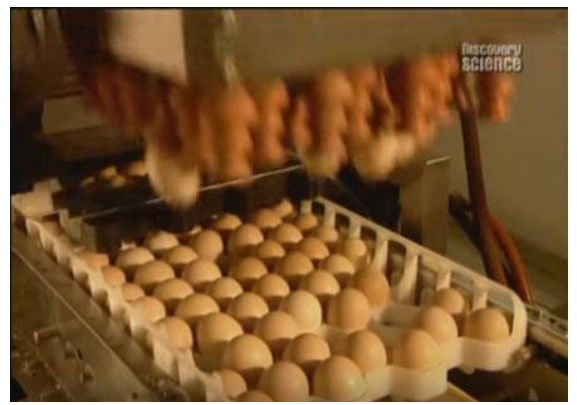

Fig. 4. Fragment of installation of Inovoject (automatic removal of eggs with dead embryos).

This section is devoted to the features of the use of microcontroller technology to the tasks of creating the first part of the installation (OVOJECTORS). This installation includes a diagnostic part (sensor block of 30 or 150 pieces), a microcontroller control unit (a multi-output microcontroller with a block of electromagnetic relays for 30 or 150 pieces), a block of mechanical extraction of eggs, which includes installation of pneumatic pick-up of objects of extraction. The latter is a device for moving a gripper block -30 of a vacuum gripper using an air supply unit for 30 chambers connected to an electromagnetic relay controlled by a microcontroller unit. The kind of working finished device is shown in Fig.1. In branded installations, a diagnostic unit with optical sensors working on the through-clearance of eggs in the supplied tray is usually integrated with an extraction device. This certainly speeds up the operation of the device, although it introduces technological difficulties in the production of this product. As it imposes additional conditions on the parameters of the sensors, but it saves money on the absence of the need to create a separate panel on which the sensors are placed and there is no need to position it.

\section{DEVISES FOR CIS PROPOSITION}

When the positioning of the tray with eggs is completed, the microcontroller automatically, in a cycle, starts polling infrared sensors that see through all 30 (150) eggs [4.5]. In the case of a signal level below the threshold value (which corresponds to an egg and a dead embryo), the number of the activated sensor is recorded in the corresponding EEPROM (memory) cell of the microcontroller's memory. At the end of the survey, the signals from the inputs of the microcontroller connected to the electromagnetic relay with optical decoupling include pneumatic devices that extract eggs with a dead embryo.

This happens as follows. Upon completion of recording the result of polling the sensors in the EEPROM memory of the microcontroller, a delay is set, after which the process of polling the EEPROM memory begins. Starting from a known starting address, for example, $00001 \mathrm{H}$, the contents of the cells in the loop are read to the address, for example, $00029 \mathrm{H}$. Each cell is programmed to correspond to a device for pneumatic extraction of eggs with dead embryos. Totally there are 30 or 150 pieces, as well as the number of infrared sensors. With 150 sensors for their control, in addition to the microcontroller, decryption devices are used, since the number of digital outputs of a typical inexpensive microcontroller is limited. When receiving information from the EEPROM memory cell corresponding to the object to be retrieved, the microcontroller through the terminals connected to the actuators through the relay brings the pneumatic gripping device to the corresponding object. This happens until the reading cycle of the EERROM memory cells ends. General view of the working installation is shown in Fig. 4.

This process is also controlled by a computer connected to the microcontroller via standard interfaces (USB, etc.) It is possible to modify the wireless interface to transmit information to a computer using the GSM-sim800 module, as well as Wi-fi, Blootuze modules. Installing a local server will make it possible to monitor the results of sorting eggs with live and dead embryos in On-line mode in the local network of the enterprise. It is also possible to transfer data to the Internet.

Features of the development and use of the installation in the conditions of the CIS and Ukraine. Despite the economic feasibility from the point of view of increasing labour productivity in poultry enterprises, the production of a full complex (Ovojectors) is not always appropriate. The reason for this is the high cost of maintenance, as well as the inevitable dependence on imported components.

\section{CONCLUSIONS}

Work on the development of devices for the automation of processes for the removal of eggs with dead embryos and in ovo vaccination abroad are closed and publications do not exist. Thus, Embrex (USA) is offering a vaccination system for rent in more than 30 countries in Europe, Latin and North America, Australia, and Asia. Embrex does not sell machines and licenses for its production. Based on the experimental and design work carried out in Ukraine, it is quite possible to create industrial devices for the automatic removal of eggs from inert living embryos and to further in ovo vaccinate live embryos, which will be completed domestic poultry enterprises. In the world, there is an analogue, which was created by Embrex (USA), which only leases its products for 220 thousand dollars a year for one device. Domestic automatic devices for the removal of inanimate embryos and subsequent injecting of living will be cheaper by 3-4 times for US denials. It is most expedient in domestic to produce plants separating eggs with living and dead embryos separately from the ovo vaccination block.

\section{REFERENCES}

[1] V. Breslavets and A. Stegniy, "On the Question to Development of Automatic Methods of Biocontrol in the Incubation", Veterinarna medicina, vol. 99, pp. 186-189, 2014.

[2] V. Rolnik, Biology of embryonic development of birds. Leningrad: Science, 1968, p. 425. 